\title{
昆明地区昭觉林蛙生殖及附属器官的年周期变化
}

\author{
周 伟*，李 旭，吴建普，李凤莲，李明会 \\ (西南林学院 保护生物学学院, 云南省高校森林灾害预警与控制重点实验室, 云南 昆明 650224)
}

\begin{abstract}
摘要: 解剖2006年4-12月自昆明东北郊采集到的体长大于 $40.0 \mathrm{~mm}$ 的昭觉林蛙(Rana chaochiaoensis)标本73只 ( 939,334$)$, 测量其体长、体重、肝重、脂肪体重、卵巢重、精巢重、输卵管重和直径、卵径等。以体长(或体重) 为协变量做协方差分析(ANCOVA), 结果显示, 输卵管直径、卵径, 雌性的肝重、脂肪体重、卵巢重和输卵管重 和雄性的精巢重在年周期内差异极显著。相关性检验显示, 雌性卵巢重分别与肝重、输卵管重、输卵管直径、卵 径均显著正相关, 即它们的发育完全同步, 但与脂肪体重不相关。各月雌体卵巢重和雄体精巢重的发育极显著正 相关，即雄性与雌性的性腺发育同步。据解剖、检验分析及野外观察结果分析证明，昆明地区昭觉林蛙的繁殖时 间在10月完成，且一年只有一个繁殖期。脂肪体在雌性个体生殖腺发育过程中起供能作用。在卵巢发育过程中， 肝供能作用不明显, 但在雌性个体抱对和产卵过程中起供能作用。在雄性性腺发育的过程中, 肝和脂肪体的供能 作用不如䧳性明显, 耗能极低。可见昭觉林蛙雄性个体的生殖投入少于雌性个体。一些雌性个体推迟冬眠时间, 主要是为受食和补充能量, 以便安全越冬。此外, 新生昭觉林蛙蝌蚪期在冬季度过, 随后的发育和变态面临旱季 缺水环境。所以, 加强对昭觉林蛙产卵高峰期、蝌蚪越冬期和渡过干旱缺水期等生活周期中脆弱环节的环境保护, 有利于种群的延续。
\end{abstract}

关键词：昭觉林蛙；生殖器官；脂肪体；年周期变化

中图分类号: Q959.5; Q492 文献标识码: A 文章编号: 0254-5853-(2008)01-0089-06

\section{Annual Changes in Reproductive and Accompanying Organs of Rana chaochiaoensis in the Kunming Area}

\author{
ZHOU Wei ${ }^{*}$, LI Xu, WU Jiang-pu, LI Feng-lian, LI Ming-hui \\ (Faculty of Conservation Biology, Southwest Forestry College; Key Laboratory of Forest Disaster Warning and Control in \\ Yunnan Higher Education Institutions, Kunming 650224, China)
}

\begin{abstract}
Seventy-three specimens $(\$ 39, \precsim 34)$ of Ranna chaochiaoensis whose snout-vent length was more than $40 \mathrm{~mm}$ were collected during April to December of 2006 from a northeast suburb of Kunming. Their snout-vent length, body weight, liver and body fat, weight of ovary and spermary, weight and diameter of oviduct, and diameter of egg measurements were taken. The results were analyzed by ANOVA with the length of the snout-vent (or weight of body) as the covariate factor, showed that there were significant differences in the diameter of the oviduct and egg; weight of liver, body fat, ovary and oviduct in females and weight of spermary in males. The results of the correlation test showed that weight of ovaries had a significant positive correlation with the weight of the liver and oviduct, diameter of oviduct and eggs in females but not with the weight of body fat, which meant that the development of ovary weight corresponded with the development of these organs. The development of ovaries and spermary weight had significant positive correlation in each month, which meant that the development of male sex glands synchronized with that of females. According to the results of dissections, analysis and field observation in the Kunming area, the reproductive time of $R$. chaochiaoensis finished in October, with an annual reproductive peak. Body fat could supply energy during the development process of sex glands in females. During the developmental process of ovaries, the liver did not supply energy; however, it supplied energy during the process of amplexus and spawning. Compared with females, in males the effect of supplying energy from the liver and body fat was evident during the development process of the sex glands, which meant that little energy was expended. So the reproductive investment of males was less than that of females. Some individual females put off hibernation so they could feed and increase their energy through winter. However, it would cause mortality in adults as the temperature drops dramatically. Newborn tadpoles of $R$. chaochiaoensis would
\end{abstract}

收稿日期: 2007-10-26; 接受日期: 2007-11-26

基金项目：云南省林业厅“云南省珍稀濒危动植物及栖息地、保护区的救护繁育项目”(200619) 资助

”通讯作者 (Corresponding author), E-mail: weizhouyn@163.com 
also face a desolate environment during winter and lack resources during their development and metamorphosis. It would be beneficial for the continuance of the population to protect the environment where $R$. chaochiaoensis lives during the rothole linked with their life cycle, such as the peak of spawning, hibernation and the dry season when tadpoles develop.

Key words: Rana chaochiaoensis; Reproductive organ; Body fat; Annual changes

无尾两栖类抱对、鸣叫和产卵等繁殖行为出现 在相对固定的时间内。雌性个体的卵巢、输卵管和 卵等生殖器官和组织细胞的变化呈现稳定的周年 变化规律(Redshaw, 1972; Zhang et al, 1985), 雄性个 体的精巢亦有年周期变化的现象(Yang \& Zhang, 2005)。生殖腺和生殖细胞及其附属器官发育程度的 变化状态可成为研究繁殖周期的指标。目前的相关 研究多是对两性生殖腺、生殖细胞及其附属器官的 形态变化的定性描述, 或亚显微结构、激素等的周 期变化的观察(Wang \& Zhang, 2004), 对其生殖腺、 生殖细胞及其附属器官形态的系统度量比较和相 关性分析的研究少见报道, 亦未见两性间上述内容 的比较研究。

当两栖类处于极度饥饿的状态下，或刚完成繁 殖时, 脂肪体中的能量物质明显降低(Carlo et al, 1996)。两栖类肝脏中的糖元含量有稳定的年周期变 化规律, 在越冬过程中糖元消耗尤为突出(Barni \& Bernocchi, 1991; Fenoglio et al, 1992)。宏观形态和 微观结构的证据证明, 脂肪体和肝脏是两栖类生活 史中重要的能量存储组织。检验它们在繁殖过程中 与两性性腺发育状态的相关性, 将为研究两性生殖 投入差异提供证据。

昭觉林蛙(Rana chaochiaoensis Liu)广泛分布于 林缘和农耕地, 对该蛙的生态学研究多集中在形态 描述、生态习性观察和食性分析等方面 (Zhou et al, 2003; Yang et al, 2006), 至今未见有关繁殖的专门研 究。本研究拟量化比较昆明地区昭觉林蛙生殖腺和 生殖细胞及其附属器官在一年中的发育情况, 检验 卵巢、精巢、输卵管和卵等度量因子间发育的同步 程度, 并分析生殖器官发育时间和繁殖行为出现时 间之间的相关性, 将有助于研究该蛙的繁殖起止时 间和持续期限, 一年中的繁殖次数以及两性生殖投 入是否存在差异。

\section{1 材料与方法}

\section{1 标本采集及测量}

标本采集于昆明市东北郊昭觉林蛙分布较为 集中的呼马山和小弯山。采集时间为 2006 年 1-12 月, 每月采集 2-3 次, 每月的标本中均含雌雄成 体。共 73 只(939, 万34), 均是体长大于 $40.0 \mathrm{~mm}$ 的性腺成熟个体(Yang et al, 2006)。2006 年 1-3 月 和 2007 年 1 月没有采集到标本, 因此期正值昆明 地区昭觉林蛙的冬眠期。而 2006 年 11-12 月尽管 加大了采集力度, 标本数量依然偏少, 因蛙类已逐 渐进入冬眠。所获标本用 $5 \%$ 的甲醛溶液浸泡, 保 存待用。

用游标卡尺(天平 $\mathrm{I}$ 型， 0- $150 \mathrm{~mm}$ ) 测量体长(精 度 $0.1 \mathrm{~mm}$ ), 在SMZ645型解剖镜 (最大精度为 0.02 $\mathrm{mm})$ 下测量输卵管直径 $(\times 20$ 倍 $)$ 和卵径 $(\times 50$ 倍 $)$ 。随机 选择解剖镜视野中相连的 30 个卵, 测量它们的直径 后取其平均值为卵径, 以减小测量误差。用 ALC-1100.2型电子分析天平(精度 $0.01 \mathrm{~g}$ )称量体重, 用BS223S型电子分析天平 (精度为 $0.001 \mathrm{~g}$ ) 分别称 量肝、脂肪体、卵巢、输卵管和精巢重。

\section{2 数据分析}

用Kolmogorov Smirnov Z-检验雌雄蛙的生殖 腺、生殖细胞、肝和脂肪体数据的正态性。当数据 符合正态分布时, 采用Pearson相关性检验; 否则采 用非参数Spearman相关性检验。用相关性检验分析 雌(或雄)蛙体长(或体重)与生殖腺、生殖细胞及储能 组织(如体重和卵巢重、卵径和体长)等数据间的相 关性(Yu \& He, 2003)。对与体长(或体重)无显著相关 $(P \geqslant 0.05)$ 的变量, 采用单因素方差分析(One-way ANOVA) 检验各月雌(或雄)蛙生殖腺和储能组织等 变量是否存在显著性差异; 否则, 用体长(或体重) 为协变量, 做协方差分析(ANCOVA), 检验各月雌 (或雄)蛙生殖腺和储能组织等变量是否存在显著性 差异, 以排除体长(或体重)对统计结果的影响。选 出随月份变化而呈现显著性差异的因子, 检验各因 子间可量数据的相关性, 以验证雌、雄蛙性腺发育 是否同步, 或者分析雌、雄生殖投入的差异。数据 统计在SPSS 13.0 for Windows和Excel 2003软件中 完成。 


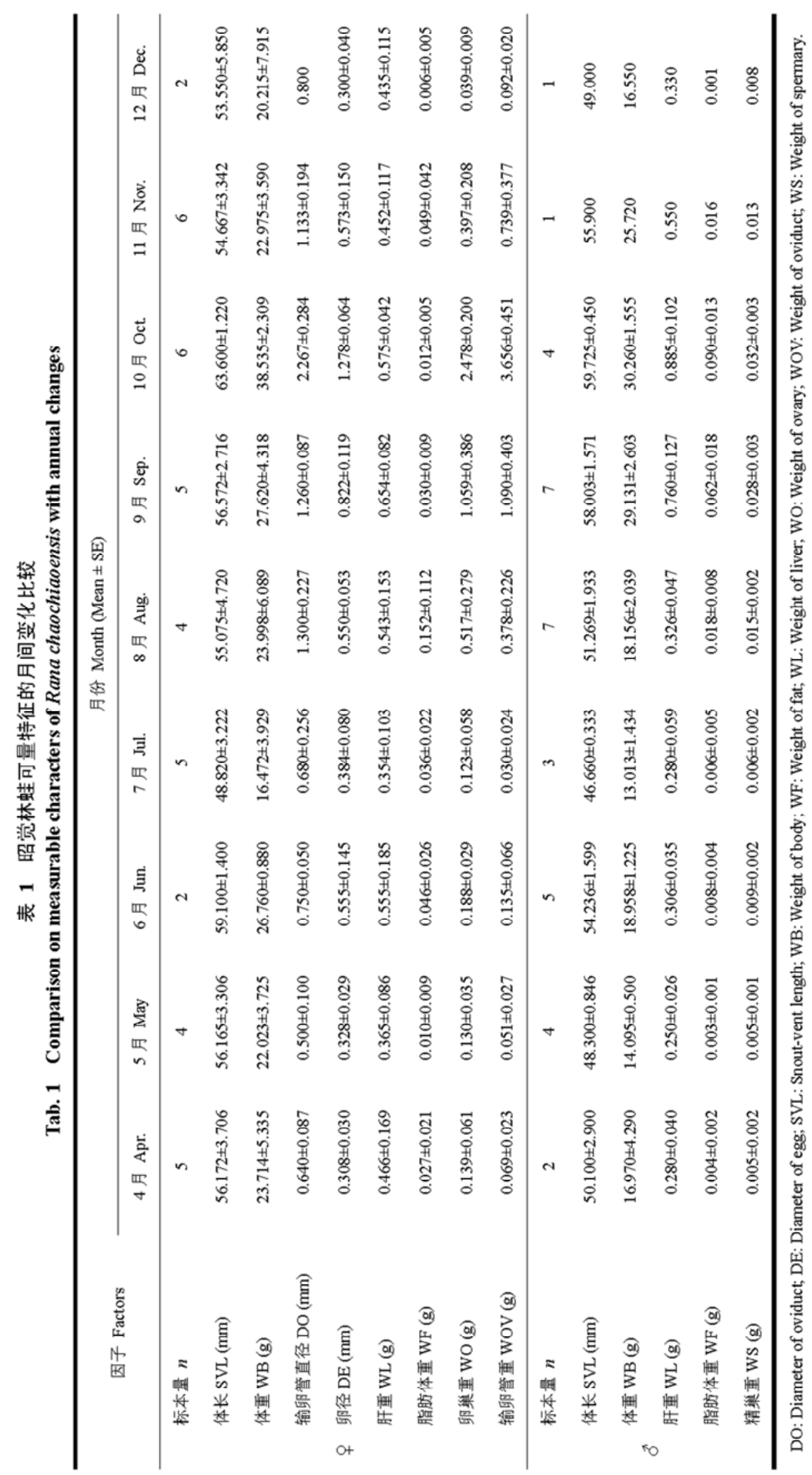




\section{2 结果与分析}

\section{1 雌性器官的年变化}

输卵管直径和卵径至10月达到最大值, 呈现显 著的发育高峰期。肝重在 9 月为最大。脂肪体重在 8 月达最大值, 而至 10 月降至最小值, 在一年中亦有 一高峰期。输卵管直径和卵径的峰值出现时间与卵 巢和输卵管重的峰值出现时间吻合, 惟有脂肪体重 的峰值出现较早(表1)。

经Kolmogorov Smirnov $Z$-检验, 雌性昭觉林蛙 个体的体长 $(P=0.495)$ 、体重 $(P=0.378)$ 、肝重 $(P=0.186)$ 和输卵管直径 $(P=0.053)$ 的数据呈正态分 布; 脂肪体重 $(P=0.001)$ 、卵巢重 $(P=0.003)$ 、输卵管 重 $(P=0.001)$ 和卵径 $(P=0.021)$ 的数据不呈正态分布。

体长与输卵管直径 $(r=0.515, P<0.001, n=39)$ 、卵 径( $r=0.525, P<0.001, n=39)$ 分别呈极显著正相关, 故 以体长为协变量作协方差分析。结果显示, 输卵管 直径和卵径的变化随月份变化具显著性差异(表2)。

体重分别与肝重 $(r=0.826, P<0.001, n=39)$ 、脂肪 体重 $(r=0.484, P=0.002, n=39)$ 、卵巢重 $(r=0.687$, $P<0.001, n=39)$ 和输卵管重 $(r=0.714, P<0.001, n=39)$ 极显著正相关, 故以体重为协变量作协方差分析。 结果显示, 雌性肝重、脂肪体重、卵巢重和输卵管 重的变化随月份的变化具显著性差异(表 2)。

随月份变化呈现显著差异的各项因子中, 卵巢 重分别与肝重 $(r=0.555, P<0.001, \mathrm{n}=39)$ 、输卵管重 $(r=0.846, P<0.001, n=39)$ 、输卵管直径 $(r=0.805$, $P<0.001, n=39)$ 和卵径 $(r=0.876, P<0.001, n=39)$ 极显 著正相关, 但与脂肪体重不相关 $(r=0.281, P=0.083$, $n=39$ )。

\section{2 雄性器官的年变化}

雄性蛙肝重和脂肪体重的发育比较均衡, 未出 现规律性峰值。精巢重在 10 月达最大值, 呈现显
著的发育高峰期(表 1)。

经 Kolmogorov Smirnov $Z$-检验, 雄性个体的体 长 $(P=0.554)$ 、体重 $(P=0.227)$ 、肝重 $(P=0.057)$ 和精巢 重 $(P=0.315)$ 的数据呈正态分布, 脂肪体重 $(P=0.024)$ 的数据不呈正态分布。

体重分别与肝重 $(r=0.949, P<0.001, n=34)$ 、脂肪 体重 $(r=0.779, P<0.001, n=34)$ 和精巢重 $(r=0.896$, $P<0.001, n=34)$ 呈极显著正相关, 故以体重为协变量 作协方差分析。结果显示, 雄性肝重和脂肪体重的 变化随月份的变化不具显著性差异, 但精巢重的变 化具显著性差异(表 2)。此外, 精巢重与肝重( $r=0.871$, $P<0.001, n=34)$ 、脂肪体重 $(r=0.769, P<0.001, n=34)$ 呈极显著正相关。

\section{3 雌、雄器官的同步性比较}

因昭觉林蛙雌雄两性的脂肪体重、卵巢重和精 巢重与体重显著相关, 为消除体重的影响, 将每一 个体的脂肪体指数、卵巢指数、精巢指数作非参数 Spearman 相关性检验。检验结果表明, 昭觉林蛙雌 雄两性各月间的脂肪体重不相关 $(r=0.008, P=0.983$, $n=9)$, 但雌体卵巢重和雄体精巢重呈极显著正相关 $(r=0.812, P=0.008, n=9)$ 。

\section{3 讨 论}

\section{1 繁殖时间与繁殖次数}

卵巢重量的增加与卵的发生及发育相关; 当输 卵管的直径和重量增加到最大值时, 则是该种蛙类 繁殖的标志。昭觉林蛙的卵巢重量、输卵管直径和 重量自 8 月开始发育, 10 月达到最大值, 11 月降 至最小值。雄性的精巢重自 8 月开始增加, 11 月降 低(表 1)。数据协方差分析结果表明, 昭觉林蛙的输 卵管直径和卵径的变化随月份变化具显著性差异, 且精巢重的变化亦随月份的变化具显著性差异(表 2)。而且雄性精巢与雌性卵巢的发育在时间上显著

表 2 与体重和体长呈显著相关性特征的协方差分析结果

Tab. 2 The results by ANCOVA on some characters with significant correlation related to body weight and length

\begin{tabular}{|c|c|c|c|c|c|c|c|}
\hline \multirow{2}{*}{ 因子 Factors $(q)$} & \multicolumn{3}{|c|}{ 协方差分析 ANCOVA } & \multirow{2}{*}{ 因子 Factors (ठ) } & \multicolumn{3}{|c|}{ 协方差分析 ANCOVA } \\
\hline & $F$ & $d f$ & $P$ & & $F$ & $d f$ & $P$ \\
\hline \multicolumn{8}{|c|}{ 以体重为协变量 Weight of body as covariant factor } \\
\hline 肝重 WL (g) & 3.859 & 8,38 & $0.003^{* *}$ & 肝重 WL (g) & 2.159 & 8,33 & 0.069 \\
\hline 脂肪体重 WF (g) & 3.816 & 8,38 & $0.004^{* *}$ & 脂肪体重 WF (g) & 1.234 & 8,33 & 0.322 \\
\hline 卵巢重 WO (g) & 8.703 & 8,38 & $<0.001^{* *}$ & 精巢重 WS (g) & 4.180 & 8,33 & $0.003^{* *}$ \\
\hline 输卵管重 WOV (g) & 8.988 & 8,38 & $<0.001^{* *}$ & & & & \\
\hline \multicolumn{8}{|c|}{ 以体长为协变量 Length of snout-vent as covariant factor } \\
\hline 输卵管直径 DO (mm) & 5.917 & 8,38 & $<0.001^{* *}$ & & & & \\
\hline 卵径 DE (mm) & 8.832 & 8,38 & $<0.001^{* *}$ & & & & \\
\hline
\end{tabular}

缩写同表 1 (Abbreviation is the same as Tab. 1)。 ${ }^{*} P<0.05,{ }^{* *} P<0.01$. 
相关, 卵巢、输卵管和卵发育完全同步, 即当雌性 的生殖器官开始为产卵做准备时, 雄性的生殖器官 亦开始为授精做相应的准备。所以, 10 月应为昭觉 林蛙主要的产卵时期, 11 月基本停止产卵。据野外 观察结果, 10 月昭觉林蛙出现抱对行为, 并观察到 卵团。而 11 月再未观察到抱对, 证实繁殖行为在 11 月时已经停止。解剖结果与野外生态观察结果基 本吻合, 说明昆明地区昭觉林蛙的繁殖时间在 10 月, 且一年只有一个繁殖期。

\section{2 两性生殖投入的差异}

如果肝和脂肪体的重量周年变化与生殖腺的 变化存在某种相关性, 则反映它们对生殖腺提供能 量。因为两栖类肝和脂肪体是重要的能量储存组织 (Barni \& Bernocchi, 1991; Fenoglio et al, 1992; Carlo et al, 1996)。

脂肪体在昭觉林蛙的雌性个体生殖腺发育中 起到提供能量的作用。解剖和检验结果表明, 雌性 昭觉林蛙卵巢发育与脂肪体的变化不同步, 且脂肪 体重、卵巢重和输卵管重的变化随月份的变化具显 著性差异。测量数据表明, 卵巢自 8 月起开始发育, 此时脂肪体重为最大值; 10 月为卵巢发育的高峰 期, 但脂肪体在此时的重量降至最小值(表 1), 说明 雌性性腺在发育的时候, 脂肪体逐渐被消耗。对中 华蟾蜍(Bufo gargarizans) 的研究结果也如此(Zhou \& Sun, 1997)。

肝在昭觉林蛙雌性个体抱对和产卵过程中到 了提供能量的作用。肝重在 9 月为最大值, 而卵巢 在 10 月为发育的高峰期(表 1)。卵巢在 11 月为最小 值, 而肝在 11 月的重量显著低于 10 月时的重量(表 1)。暗示肝中的能量物质在昭觉林蛙雌性个体发生 抱对和产卵等行为的过程中被消耗, 即肝在雌性个 体抱对和产卵过程中起到了提供能量的作用。检验 结果表明, 卵巢发育与肝的变化同步, 即在卵巢发 育过程中, 肝提供能量的作用不如在繁殖过程中的 作用明显。

昭觉林蛙雄性个体的肝和脂肪体的能量损耗 比雌性的低。雄性的肝重和脂肪体重与体重呈极显 著正相关, 即肝重和脂肪体重受雄性个体体重的变 化影响, 但肝重和脂肪体重的变化随月份的变化却

\section{参考文献:}

Barni S, Bernocchi G.. 1991. Internalization of erythrocytes into liver parenchymal cells in naturally hibernating frogs (Rana esculenta L.) [J]. Journal of Experimental Zoology, 258: 143-150.
不具显著性差异(表 2), 即雄性的肝和脂肪体在一年 中发育均衡。此外, 精巢重与肝重和脂肪体重呈极 显著的正相关，而不是负相关。所有的结果均提示， 在雄性个体性腺发育的过程中, 肝和脂肪体的能量 提供作用不如雌性明显, 或者说伴随着雄性的性腺 发育, 肝和脂肪体的能量损耗极低。所以, 昭觉林 蛙雄性个体的生殖投入少于雌性个体。

\section{3 生殖规律与保护措施}

加强昭觉林蛙蝌蚪越冬水域的管理。昭觉林蛙 的繁殖高峰集中在 10 月, 其后代以蝌蚪形式越冬, 并于次年 5 月完成变态和登陆(Zhou et al, 2005)。此 期间, 蝌蚪都生活在有水的地方。蝌蚪变态期正值 昆明的旱季, 一些池塘和临时性水塘, 水域面积相 对较小, 极少有补水机会或者补水量较小。而随着 季节的变化和时间的推移, 温度上升, 蒸发量加大。 如果这些水域面积较小的水塘干涸, 势必影响水塘 里大部分蝌蚪的变态, 对该种的延续构成威胁。此 外, 对无尾两栖类一些蚪蚪的急性毒性研究表明, 蝌蚪对于农药的耐受性较低(Michelle et al, 2005)。 因此在 10 月至次年 5 月这段时间, 加强水域环境 的管护, 避免水域干涸和受污染, 这对于昭觉林蛙 蝌蚪的发育至关重要。

注重雌性繁殖个体的保护。昆明地区两栖类动 物多数一般在 12 月已进入冬眠, 但昭觉林蛙在水 草地的活动一直可以持续到 12 月底, 尤其是雌性。 因为昭觉林蛙雌性个体的生殖投入较雄性的高。推 迟冬眠时间主要是为了受食和补充能量, 以便能够 安全越冬。因此, 如果在 12 月遇上低温天气, 或 者在翌年初碰上低温和下雪, 能量补充不足的雌性 成蛙和蝌蚪均难以生存。这乃是导致昭觉林蛙种群 数量年间波动较大的主要原因。

注重珍稀濒危动物生命周期中的脆弱环节的 研究。虽然昭觉林蛙不属于珍稀濒危种类和国家保 护动物, 但对其生活史的研究和分析结果提示, 对 产卵高峰期、越冬期和干旱缺水期等环节进行有效 管理, 对该物种种群的延续和个体数量的增加致关 重要。有鉴于此, 如果对一些珍稀濒危种类加强生 活史的研究, 了解其生命周期中的脆弱环节, 对制 定保护计划和措施将极具参考价值。 
Fenoglio C, Bernocchi G, Barni S. 1992. Frog hepatocyte modifications induced by seasonal variations: A morphological and cytochemical study [J]. Tissue Cell, 24: 17-29.

Michelle DB, Christine MB, James FF, Edward EL. 2005. Multiple sublethal chemicals negatively affect tadpoles of the Green Frog, Rana clamitans [J]. Environmental Toxicology and Chemistry, 24 (5): 1267-1272.

Redshaw MR. 1972. The hormonal control of the amphibian ovary [J]. American Zoologist, 12: 289-306.

Wang HY, Zhang YH. 2004. Annual cycle of testicular microstructures in stream salamanders (Batrachuperus tibetanus) [J]. Zool Res, 15 (6): 484-490. [王宏元, 张育辉. 2004. 北方山溪鲵精巢显微结构的年 周期变化. 动物学研究, 15 (6): 484-490.]

Yang Y, Zhou W, Li X, Pan XF. 2006. Diet and sexual dimorphism of Rana chaochiaoensis [J]. Journal of Zhejiang Forestry College, 23(5) : 560-564. [杨颖, 周伟, 李旭, 潘晓赋. 2006. 昭觉林蛙的食性与两 性异形. 浙江林学院学报, 23 (5) : 560-564.]

Yang C, Zhang YH. 2005. Annual variation of the testicular microstructure of Rana chensinensis in the north slope of Qinling Mountains [J]. Chinese Journal of Zoology, 49(3): 81-85. [杨纯, 张育辉. 2005. 秦 岭北坡中国林蛙精巢显微结构的年周期变化. 动物学杂志, 40(3): 81-85.]
Yu JY, He XH. 2003. Statistics and analysis for data the application of SPSS [M]. Beijing: Post \& Telecommunications Press. [余建英, 何旭宏. 2003. 数据统计与 SPSS 应用. 北京: 人民邮电出版社.]

Zhang J, Liu J R, Cai M Z. 1985. A study on the seasonal variation in the ovary and the reproductive frequency in Rana limnocharis [J]. Acta Herpetologica Sinica , 4 (4): 267-275. [张健, 刘俊仁, 蔡明章. 1985. 泽蛙卵巢季节性变异及生殖频率的研究. 两栖爬行动物学 报, 4 (4): 267-275.]

Zhou HM, Sun JM. 1997. The seasonal rhythm of fat content in fat-body of Chinese toad [J]. Sichuan Journal of Zoology, 16(2): 95-96. [周化民, 孙建梅. 1997. 大蟾蜍脂肪体脂肪的季节变化. 四川动物, 16(2): 95-96.]

Zhou W, Li MH, Pan XF. 2003. Morphological differentiation between Rana pleuraden and Rana chaochiaoensis with comments on their potential adaptive significance [J]. Zool Res, 24 (6): 445-451. [周伟, 李明会, 潘晓赋. 2003. 滇蛙和昭觉林蛙的形态差异及其潜在的 适应意义. 动物学研究, 24 (6): 445-451.]

Zhou W, LI MH, Zhang XY, He JF. 2005. Food comparison between tadpoles of Rana catesbeiana and $R$. chaochiaoensis collected from the same habitat[J]. Zool Res, 26 (1): 89-95. [周伟, 李明会, 张兴宇, 贺佳飞. 2005. 同一生境牛蛙与昭觉林蛙蝌蚪的食性比较. 动物 学研究, 26 (1): 89-95.]

\section{（上接第 77 页）}

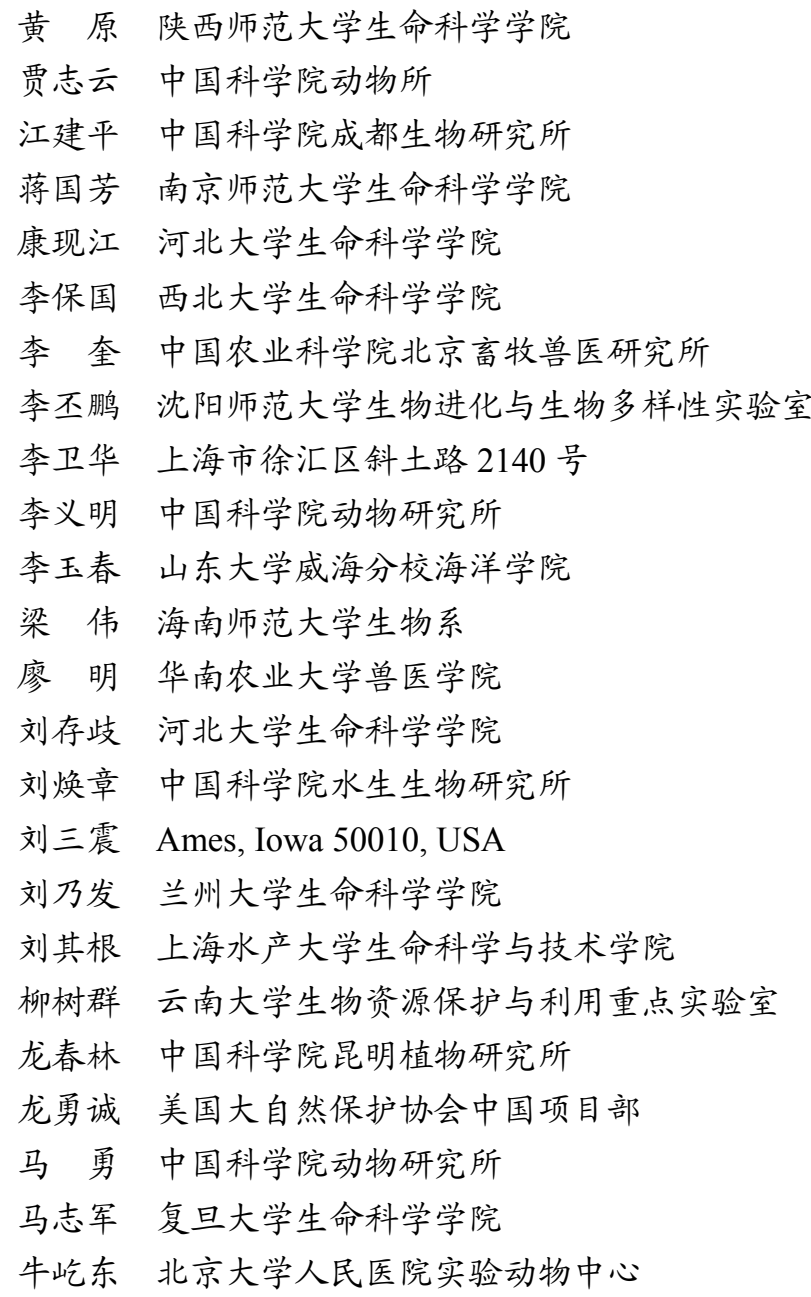

秦川中国医学科学院实验动物研究所

石建斌中国林科院森林生态环境与保护研究所

史海涛 海南师范大学生物系

宋延龄 中国科学院动物研究所

郃发道陕西师范大学生命科学学院

唐文乔上海水产大学鱼类研究室

童春富华东师范大学河口海岸学国家重点实验室

汪 松 中国科学院动物研究所

王安利华南师范大学生命科学学院

王金星山东大学生命科学学院

王小明 华东师范大学生命科学学院

王义权 厦门大学生命科学学院

王跃招中国科学院成都生物研究所

魏辅文中国科学院动物研究所

吴峎河北大学生命科学学院

吴孝兵安徽师范大学生命科学学院

吴应积蒙古大学哺乳动物生殖生物学及生物技术室

武正军广西师范大学生命科学院

向余劲攻 复旦大学生命科学院

谢 锋 中国科学院成都生物研究所

徐盈中国科学院水生生物研究所生态毒理组

许木启 中国科学院动物研究所

薛俊增 杭州师范大学生命与环境科学学院

杨卫军 杭州市浙江大学紫金港校区生命科学学院 